\title{
Hypersonic Wake Diagnostics Using Laser Induced Fluorescence Techniques
}

\author{
Jack L. Mills* and Charles I. Sukenik ${ }^{\dagger}$ \\ Old Dominion University, Department of Physics, Norfolk, Virginia, 23529 \\ Robert J. Balla $\ddagger$ \\ NASA Langley Research Center, Hampton, Virginia, 23681
}

\begin{abstract}
A review of recent research performed in iodine that involves a two photon absorption of light at $193 \mathrm{~nm}$ will be discussed, and it's potential application to velocimetry measurements in a hypersonic flow field will be described. An alternative seed atom, Krypton, will be presented as a good candidate for performing nonintrusive hypersonic flow diagnostics. Krypton has a metastable state with a lifetime of approximately $43 \mathrm{~s}$ which would prove useful for time of flight measurement (TOF) and a sensitivity to collisions that can be utilized for density measurements. Calculations using modest laser energies and experimental values show an efficiency of excited state production to be on the order of $10^{-6}$ for a two photon absorption at $193 \mathrm{~nm}$ to the $6 p[3 / 2]_{2}$ state.
\end{abstract}

\section{Introduction}

Understanding the gas dynamics of compressible flow fields becomes complicated as the change in the initial flow parameters with respect to the regions of interest, like the wake region, become quite large. Traditional methods of measuring important aerothermodynamic quantities like velocity, temperature, and density are intrusive and can alter flow dynamics, which has led to the motivation for developing nonintrusive measurements. The need to develop non-intrusive flow diagnostics is not a new problem, the most utilized technique being laser-induced fluoresence (LIF). Using LIF Miles ${ }^{1}$ and Zimmerman ${ }^{2}$ successfully used sodium to make velocimetry, pressure, and temperature measurements in a hypersonic helium flow field. The structure of the Sodium atom is well understood and the dipole transitions of an atomic species are quite strong; however, it oxidizes very quickly in air and violently reacts with water vapor. Like all alkali metal atoms this would make it undesirable for making measurements in air. The need for a seed atom, or molecule, that is non reacting and fairly well understood has led to a lot of recent research using iodine. Much of the early research has focused on LIF using the $514.5 \mathrm{~nm}$ line of an Argon-ion laser. Flow visualization, ${ }^{3}$ velocity, ${ }^{4-7}$ pressure,${ }^{7,8}$ temperature, ${ }^{9}$ and density ${ }^{4,10}$ measurements have been made in flow fields using LIF of iodine with varying degrees of success. ${ }^{11}$ Hiller and McDaniel ${ }^{5}$ achieved an estimated accuracy of $\pm 10 \%$ when measuring velocity in subsonic gas flows. Hiller and Hansen ${ }^{7}$ achieved errors of around $6 \%$ for both pressure and velocity measurements.

Iodine makes the following transition, $B\left[{ }^{3} \Pi_{u}^{+}\right] \leftarrow X\left[{ }^{1} \Sigma_{g}^{+}\right]$, using the $514.5 \mathrm{~nm}$ Argon-ion laser line. A disadvantage of this excitation is that the $X\left[{ }^{1} \Sigma_{g}^{+}\right]$ground state is a singlet state and the $B\left[{ }^{3} \Pi_{u}^{+}\right]$excited state is a triplet state. The excitation from a singlet to triplet state is not spin allowed and makes it a weakly allowed transition, this will result in a lower fluorescence yield. Another disadvantage is a large percentage of the $B$ state population predissociates, ${ }^{12}$ which will also lead to a lower fluorescence yield. In order to get a reasonably large fluorescence signal a large quantity of iodine would need to be seeded, but due its mild toxicity and corrosiveness seeding large quantities isn’t feasible.

\footnotetext{
* Graduate Student

${ }^{\dagger}$ Professor, Physics Dept., Old Dominion University, Norfolk VA.

¥Senior Research Scientist, Advanced Sensing and Optical Measurement Branch, MS 493, Adjunct Professor, Physics Dept., Old Dominion University, Senior Member AIAA. Email: robert.j.balla@nasa.gov
} 
In an effort to improve signal levels Balla and Exton ${ }^{13,14}$ explored UV excitation, using an ArF excimer laser at $193 \mathrm{~nm}$, as an alternative to visible excitation of iodine. There is a much stronger spin allowed transition from the ground state to the excited $D\left[{ }^{1} \Sigma_{u}^{+}\right]$state (Cordes bands), which have absorption cross sections orders of magnitude larger than that of the $X$ to $B$ transition. Balla and Exton cleverly utilized the fact that decay from the $D\left[{ }^{1} \Sigma_{u}^{+}\right]$state is strongly dependent on buffer gas pressure. As the buffer gas pressure increases the population of the $D$ state is transferred to the $D^{\prime}\left[{ }^{3} \Pi_{2 g}\right]$ state, which means the fluoresence from the $D$ state at around $321 \mathrm{~nm}$ decreases and the fluoresence from the $D^{\prime}$ state at around $340 \mathrm{~nm}$ increases. Balla and Exton showed the ratio of the signal fluorescence from the $D$ and $D^{\prime}$ states is proportional to the density of the buffer gas. They found this method for determining density works quite well for pressures $>1$ Torr, but for pressures $<1$ Torr, inaccuracies are large. The $X$ to $D$ transition also suffers from signal level problems above 10 Torr when making temperature measurements.

Velocimetry measurements using iodine have only recently been performed and the preliminary results not yet published, the technique being time of flight (TOF) measurements using LIF. Recent research has become available describing a two-photon transition in iodine with $193 \mathrm{~nm}$ radiation, as opposed to the onephoton transition discussed above, which may be applicable to velocimetry measurements. The advantages this process may provide for making velocimetry measurements will be a brief subject of this paper.

The main subject of the paper will focus on an effort to remedy the limitations and drawbacks, of using iodine for making measurements of velocity, temperature, and density of hypersonic flows. This has motivated us to investigate other seed species, in particular krypton. Krypton is chemically inert, nontoxic, and its energy level structure is well understood. We will present a model for using Krypton as a seed atom for making density and time of flight (TOF) velocimetry measurements, also known as flow tagging, in a hypersonic flow field. ${ }^{15}$

\section{Iodine}

For iodine to be a well suited seed molecule it needs to be useful in making velocimetry measurements, a very important quantity for determining aerothermodynamic properties of the flow. TOF velocimetry measurements using LIF can be difficult to make depending on the seed particle. For atoms or molecules with radiative lifetimes on the order of nanoseconds; most of the fluorescence may already be gone before an image can be taken, and for low supersonic and subsonic velocities, like in the wake region of a hypersonic flow, the distance the collection of particles move may difficult to resolve. For example, in a Mach 10 flow with a radiative lifetime of $5 \mathrm{~ns}$, the atom or molecule would only move around $17 \mu \mathrm{m}$. In the wake region where velocities are thought to be around $750 \mathrm{~m} / \mathrm{s}$ (roughly Mach 2), the atom or molecule would only move around $4 \mu \mathrm{m}$. Radiative lifetimes on the order of microseconds would be more advantageous for accurate measurements in slower flows, and with typical response times of gated cameras.

Recent research has shown that a two photon transition in iodine may indeed produce states that have radiative lifetimes on the order of microseconds. It is known there is a resonant two photon transition in iodine at $193 \mathrm{~nm}$ from $B\left[{ }^{2} \Sigma_{g}^{+}\right] \leftarrow X\left[{ }^{1} \Sigma_{g}^{+}\right]$, which lies well above the atomic ionization threshold. ${ }^{16}$ Any population excited to this state will predissociate into ground state I, and ground state $\mathrm{I}^{+}$. O'Keeffe et al. ${ }^{17}$ have shown recently, that the absorption of two $193 \mathrm{~nm}$ photons can also lead to the creation of molecular Rydberg states $I^{2}\left[R\left(B^{2} \Sigma_{g}^{+}\right)\right]$. The molecular Rydberg states are considered a super excited state (SES), which is defined as a neutral excited state with internal energy greater than the lowest ionization threshold of the molecule. Upon excitation the SES will then predissociate into ground state I, and a high- $n$ Rydberg iodine atom. The lifetime of the Rydberg fragment is observed to be $325 \pm 25 \mu \mathrm{s}$, atoms with lifetimes of this order are typically in a zero kinetic energy (ZEKE) state.

Future research will focus on time dependent fluorescence measurements to determine if the long lived states radiatively decay as result of the two photon absorption, and subsequently the applicability of the ZEKE states to velocimetry measurements.

\section{Krypton}

\section{A. Proposed Experimental Technique}

Krypton may be especially well suited as a seed atom for making velocimetry measurements. There are multiple resonant two photon transitions accessible using the output of an ArF laser at $193 \mathrm{~nm}$. With the 
laser available for this experiment two fall within the gain profile, both of which can decay to the $4 p^{5} 5 s[3 / 2]_{2}$ metastable state. The $5 s[3 / 2]_{2}$ metastable state has a lifetime of approximately 28 s. ${ }^{18}$ With a radiative lifetime on the order of seconds, one can choose desired distances from the point of metastable excitation to make the velocity measurement. A way to make TOF velocimetry measurements is to create krypton metastable atoms, and further downstream excite the krypton from the metastable state to a higher excited state $5 p[5 / 2]_{3}$ and collect the resulting fluorescence at right angles to the beam. The observed wavelength of the excitation, $5 s[3 / 2]_{2} \rightarrow 5 p[5 / 2]_{3}$, is $811.29 \mathrm{~nm}$ which is within the tuning range of commercially available diode lasers. The velocity can then be calculated from the simple result

$$
v=\frac{d}{\tau_{e f f}},
$$

Where $\tau_{\text {eff }}$ would take into account delays in the camera response.

We also propose a method for measuring density based on quenching of the krypton excited states. If the quenching rates are well known, the intensity of the signal fluorescence from the $6 p[3 / 2]_{2}$ excited state will be a sensitive function of density. Future research will be performed to obtain accurate quenching rates, and determine the sensitivity of the signal fluorescence as a function of density.

\section{B. Theory}

For heavier noble gas atoms such as krypton the spin-orbit interaction $V_{s o}$ can become much larger than the residual electrostatic interaction $V_{r e}$, and the energy levels are best described by the $j l$ coupling scheme. In $j l$ coupling the orbital angular momentum $\vec{l}$ of the excited electron is strongly coupled to the total angular momentum $\vec{j}$ of the core which produces a resultant vector $\vec{k}$. The spin $\vec{s}$ of the excited electron is weakly coupled to the resultant angular momentum $\vec{k}$, which gives the total angular momentum $\vec{J}$. The terms are designated by $n l[k]_{J}$ and $n l^{\prime}[k]_{J^{\prime}}$ which correspond to the ${ }^{2} P_{3 / 2}$, and ${ }^{2} P_{1 / 2}$ ionic core states. Figure 1 shows the two sublevels of the $4 p^{5} 6 p$ electronic configuration which lie within the gain profile of the ArF laser, the $6 p[3 / 2]_{1}$ and $6 p[3 / 2]_{2}$ states.

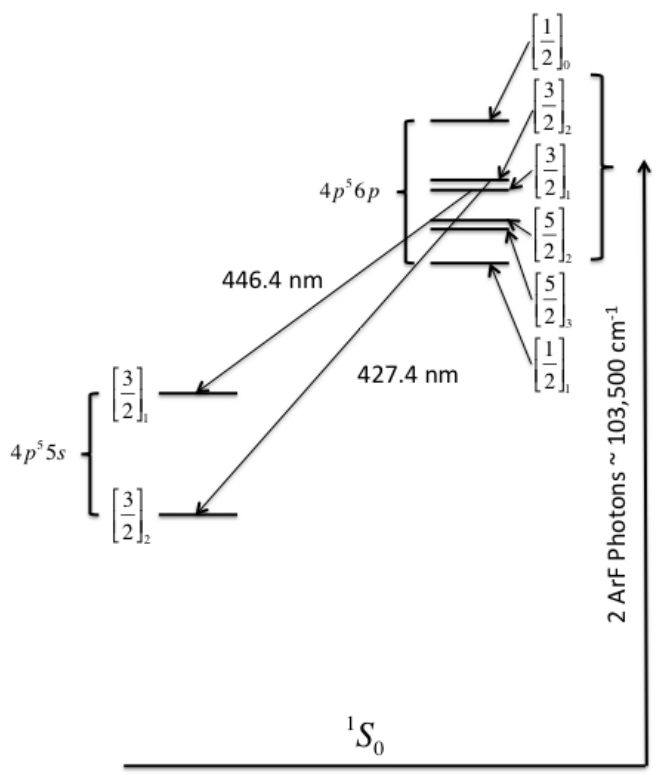

Figure 1. Partial energy level diagram for Krypton showing the $4 p^{5} 6 p$ energy levels

The two photon transition rate is given by the following expression. ${ }^{19}$

$$
W_{f g}^{(2)}=(2 \pi)^{3}(\alpha)^{2} F^{2} \omega_{L}^{2}\left|M_{f g}^{(2)}\right|^{2} g\left(\omega_{L}\right),
$$


Where $f$ and $g$ are the final and ground states, $\alpha$ is the fine structure constant, $F$ is the photon flux, $F=I / \hbar \omega, \omega_{L}$ is the angular laser frequency, $M_{f g}^{(2)}$ is the two photon matrix element, and $g\left(\omega_{L}\right)$ is the lineshape function. The two photon matrix element involves a sum over all possible channels that connect the ground and final states and is given by

$$
M_{f g}^{(2)}=\sum_{i} \frac{\langle f|z| i\rangle\langle i|z| g\rangle}{\left(\omega_{i}-\omega_{g}-\omega_{L}\right)}
$$

Where $\omega_{i}$ and $\omega_{g}$ are the angular frequency of the intermediate and ground states respectively. The lineshape function $g\left(\omega_{L}\right)$ is given by ${ }^{20}$

$$
g\left(\omega_{L}\right)=\frac{2 \sqrt{\ln (2) / \pi}}{\left[3\left(\Delta \omega_{L}\right)^{2}+\left(\Delta \omega_{T}\right)^{2}\right]^{1 / 2}},
$$

Which takes into account the bandwidth of the excitation source and the Doppler broadening of the linewidth.

The first order dipole matrix elements in Eq. (2) can be calculated from the following expression ${ }^{21}$

$$
\left|\left\langle\alpha L S J M|z| \alpha^{\prime} L^{\prime} S^{\prime} J^{\prime} M^{\prime}\right\rangle\right|^{2}=(2 J+1)\left(\begin{array}{ccc}
J & 1 & J^{\prime} \\
-M_{J} & 0 & M_{J}^{\prime}
\end{array}\right)^{2} 4.936 \times 10^{5} \frac{A}{\sigma^{3}},
$$

where $M_{J}$ is the component of the total angular momentum, $A$ is the spontaneous emission coefficient, $\sigma$ is the energy gap between states given in wavenumbers $\left(\mathrm{cm}^{-1}\right)$, and the matrix is the Wigner 3 -j symbol. The result is given in Bohr radii $\left(5.2918 \times 10^{-11} \mathrm{~m}\right)$. The output from the ArF laser in this experiment is largely unpolarized, therefore, we should expect little excitation to the $6 p[3 / 2]_{1}$ state. For linear polarization $\Delta M_{J}^{\prime}$ $=0$, we know $J^{\prime}=0$ for the Krypton ground state $\left(4 p^{6}{ }^{1} \mathrm{~S}_{0}\right)$, so it follows from the selection rules and properties of the Wigner 3 -j symbol, that $M_{J^{\prime}}=0$. For the $6 p[3 / 2]_{1}$ state $J=J^{\prime}=1$. The Wigner 3 -j symbol becomes

$$
\left(\begin{array}{lll}
1 & 1 & 1 \\
0 & 0 & 0
\end{array}\right)=0
$$

The excitation should mainly be to the $6 p[3 / 2]_{2}$ state, with maybe a little to the $6 p[3 / 2]_{1}$ state depending on the component of circular polarized light in the beam.

When calculating the two photon matrix element we use a "single path approximation", where the lowest lying accessible excited state that satisfies the angular momentum selection rules is the dominant contribution to the two photon matrix element. When using the single path approximation, the sum in Eq. (2) is reduced to one term.

$$
M_{f g}^{(2)}=\frac{\langle f|z| i\rangle\langle i|z| g\rangle}{\omega_{i}-\omega_{g}-\omega_{L}} .
$$

This result is an order of magnitude calculation, since the sum should contain all possible channels to the final state. When calculating the dipole matrix element from the ground to intermediate state we use the known oscillator strength, since the spontaneous emission coefficient has not been determined. The dipole matrix elements for the intermediate state and final state are listed in Table 1. Using these results we can calculate the two photon cross section using a laser bandwidth of $200 \mathrm{~cm}^{-1}$.

$$
\sigma_{0}^{(2)}=1.04 \times 10^{-51} \mathrm{~cm}^{4} \mathrm{~s} .
$$

To construct a rate equation analysis we calculate the photoionization cross section, since the two photon excitation is close to the ionization threshold. For a given level using the simple quantum defect approximation, which is exact for hydrogenic atoms and accurate for rare gases, the photoionization cross section is $^{22}$

$$
\sigma_{p i}=\frac{8 \times 10^{-18}}{Z \sqrt{U_{I} / R}\left(h \nu / U_{I}\right)^{3}} .
$$

We find the photoionization cross section to be $\sigma_{p i}=1.70 \times 10^{-19} \mathrm{~cm}^{2}$ 
Using the results above, taking into account ground state depletion, and neglecting collisional terms the rate equations are:

$$
\begin{gathered}
\frac{d N^{*}}{d t}=\left(N_{0}-N^{*}-N_{I}\right) \sigma_{(2)} F^{2}-N^{*} \sigma_{p i} F, \\
\frac{d N_{I}}{d t}=N^{*} \sigma_{p i} F .
\end{gathered}
$$

\section{Results}

The calculations are performed using a pulse energy of $7 \mathrm{~mJ}$, an assumed spot size diameter of $1 \mu \mathrm{m}$, a $10 \mathrm{~ns}$ pulse width, and the above calculations for $\sigma_{(2)}$ and $\sigma_{p i}$. Table 1 shows the predicted number of excited state Krypton atoms at various different pressures. These calculations predict an efficiency on the order of $10^{-6}$. We expect most of the excited state population, $6 p[3 / 2]_{2}$, to end up in the metastable state by radiative decay. Estimates of pressures in the wake region are between .5 to 2 Torr, seeding $10 \%$ would result in excited state number densities on the order of $10^{10} \mathrm{~cm}^{-3}$.

Table 1. Excited state number density as a function of pressure

\begin{tabular}{ccc}
$\begin{array}{c}\text { Pressure } \\
(\mathrm{m} \text { Torr })\end{array}$ & $\begin{array}{c}\text { Density } \\
\left(10^{15} \mathrm{~cm}^{-3}\right)\end{array}$ & $\begin{array}{c}N_{2} \\
\left(10^{10} \mathrm{~cm}^{-3}\right)\end{array}$ \\
\hline 1000 & 32.9 & 13.5 \\
700 & 23.1 & 9.45 \\
500 & 16.5 & 6.75 \\
300 & 9.88 & 4.05 \\
200 & 6.59 & 2.70 \\
100 & 3.29 & 1.35 \\
50 & 1.65 & 0.68 \\
25 & 0.82 & 0.34
\end{tabular}

\section{Conclusion}

Iodine has proven useful under certain flow conditions when making measurements of velocity, temperature, and density. The two photon excitation and subsequent creation of Rydberg states, when better understood, may prove useful for future measurements of velocity in hypersonic flow fields. Visible and UV excitation of iodine suffer from multiple signal loss mechanisms, which we hope to avoid by using Krypton. Krypton is certainly an attractive alternative since its energy level structure is well understood, its chemically inert, and nontoxic. The predicted number of excited state atoms is promising for signal level detection. Future research will consist of experimentally confirming the efficiency of metastable krypton production, and calculating quenching rates for determining sensitivity to buffer gas collisions.

\section{Acknowledgments}

This work is supported by the NASA Langley Research Center. I would like to acknowledge Dr. Charles Sukenik of Old Dominion University for graciously supplying laboratory space and equipment for future projects.

\section{References}

${ }^{1}$ R. B. Miles, "Resonant doppler velocimeter," The Physics of Fluids, vol. 18, no. 6, pp. 751-752, 1975.

${ }^{2}$ M. Zimmerman and R. Miles, "Hypersonic-helium-flow-field measurements with the resonant doppler velocimeter," $A p$ plied Physics Letters, vol. 37, no. 10, pp. 885-887, 1980. 
${ }^{3}$ K. Teshima, "Visualization for a free jet by a laser induced fluoresence method and its stucture," in Proc. Symp. Mechanics for Space Flight, pp. 52-60, 1983.

${ }^{4} \mathrm{~J}$. McDaniel, "Quantitative measurement of density and velocity in compressible flows using laser-induced iodine fluorescence," AIAA paper, no. 83-0049, 1983.

${ }^{5}$ B. Hiller, J. McDaniel, E. Rea, E. Jr., and R. Hanson, "Laser-induced fluorescence technique for velocity field measurements in subsonic gas flows," Optics Letters, vol. 8, pp. 474-476, 1983.

${ }^{6} \mathrm{~B}$. Hiller and R. Hanson, "Two-frequency laser-induced fluorescence technique for rapid velocity-field measurements in gas flows," Optics Letters, vol. 10, pp. 206-208, 1985.

${ }^{7}$ B. Hiller and R. Hanson, "Simultaneous planar measurements of velocity and pressure fileds in gas flows using laserinduced fluorescence," Applied Optics, vol. 27, pp. 33-48, 1988.

${ }^{8}$ J. McDaniel, "Nonintrusive pressure measurements with laser-induced iodine fluorescence," Prog. Astronaut. Aeronaut., vol. 92 , pp. $107-131,1984$.

${ }^{9}$ D. Fletcher and J. McDaniel, "Temperature measurement in a compressible flow field using laser-induced iodine fluorescence," Optics Letters, vol. 12, no. 1, pp. 16-18, 1987.

${ }^{10} \mathrm{~J}$. McDaniel, Investigation of laser-induced fluorescence for the measurement of density in compressible flows. PhD thesis, Stanford University, 1982.

${ }^{11} \mathrm{~B}$. Hiller and R. Hanson, "Properties of the iodine molecule relevant to laser-induced fluorescence experiments in gas flows," Experiments in Fluids, vol. 10, pp. 1-11, 1990.

${ }^{12} \mathrm{~L}$. Brewer and J. Tellinghuisen, "Quantum yield for unimolecular dissociation of $\mathrm{I}_{2}$ in visible absorption," The Journal of Chemical Physics, vol. 56, no. 8, pp. 3929-3938, 1972.

${ }^{13}$ R. J. Balla and R. J. Exton, "Density measurements in air by optically exciting the cordes bands of $\mathrm{I}_{2}$," Measurement Science and Technology, vol. 11, pp. 459-466, 2000.

${ }^{14} \mathrm{R}$. J. Balla and R. J. Exton, "Thermometry in air by optically exciting the $\mathrm{I}_{2}$ cordes bands," Measurement Science and Technology, vol. 11, pp. 1319-1327, 2000.

${ }^{15}$ P. M. Danehy, S. O'Byrne, A. F. P. Houwing, J. S. Fox, and D. R. Smith, "Flow-tagging velocimetry for hypersonic flows using fluoresence of nitric oxide," AIAA Journal, vol. 41, no. 2, pp. 263-271, 2003.

${ }^{16} \mathrm{~S}$. A. Hewitt, L. Zhu, and G. W. Flynn, "Diode laser probing of $\mathrm{CO}_{2}$ and $\mathrm{CO}$ vibrational excitation produced by collisions with high energy electrons from $193 \mathrm{~nm}$ laser photolysis of iodine," Journal of Chemical Physics, vol. 97, no. 9, pp. 6396-6409, 1992.

${ }^{17}$ P. O'Keeffe, D. Stranges, and P. Houston, "Neutral photodissociation of superexcited states of molecular iodine," The Journal of Chemical Physics, vol. 127, p. 144309, 2007.

${ }^{18}$ J. Lefers, N. Miller, D. Rupke, D. Tong, and M. Walhout, "Direct measurement of the metastable ${ }^{3} P_{2}$ decay rate in krypton," Physical Review A, vol. 66, no. 012507, 2002.

${ }^{19}$ F. H. M. Faisal, Theory of Multiphoton Processes. Plenum, 1987.

${ }^{20}$ J. Bokor, J. Y. Zavelovich, and C. K. Rhodes, "Multiphoton ultraviolet spectroscopy of some 6p levels in krypton," Physical Review A, vol. 21, no. 5, pp. 1453-1459, 1980.

${ }^{21}$ M. Weissbluth, Atoms and Molecules. Academic Press, 1978.

${ }^{22}$ N. M. Khambatta, J. A. Oertel, R. Silk, L. J. Radziemski, and J. M. Mack, "Absolute excited state and ion densities from two- and three-photon processes in some 6p levels of atomic krypton," J. Appl. Phys., vol. 64, no. 10, pp. 4809-4814, 1988. 